\title{
Improved Quantification of Bone Remodelling by Utilizing Fuzzy Based Segmentation
}

\author{
Joakim Lindblad ${ }^{1}$, Nataša Sladoje ${ }^{2}$, Vladimir Ćurić ${ }^{2}$ Hamid Sarve ${ }^{1}$, \\ Carina B. Johansson ${ }^{3}$, and Gunilla Borgefors ${ }^{1}$ \\ 1 Centre for Image Analysis, Swedish University of Agricultural Sciences, \\ Box 337, SE-751 05 Uppsala, Sweden \\ $\{$ joakim, hamid, gunilla\}@cb.uu.se \\ 2 Faculty of Engineering, University of Novi Sad, Serbia \\ \{sladoje,vcuric\}@uns.ac.rs \\ 3 Department of Clinical Medicine, Örebro University, SE-701 85 Örebro, Sweden \\ carina.johansson@oru.se
}

\begin{abstract}
We present a novel fuzzy theory based method for the segmentation of images required in histomorphometrical investigations of bone implant integration. The suggested method combines discriminant analysis classification controlled by an introduced uncertainty measure, and fuzzy connectedness segmentation method, so that the former is used for automatic seeding of the later. A thorough evaluation of the proposed segmentation method is performed. Comparison with previously published automatically obtained measurements, as well as with manually obtained ones, is presented. The proposed method improves the segmentation and, consequently, the accuracy of the automatic measurements, while keeping advantages with respect to the manual ones, by being fast, repeatable, and objective.
\end{abstract}

\section{Introduction}

The work presented in the paper is a part of a larger study aiming at improved understanding of the mechanisms of bone implants integration. The importance of this research increases together with the increased ageing of population, introducing its specific needs, which has become a characteristics of developed societies. Currently, automatic methods for quantification of bone tissue growth and modelling around the implants are in our focus. Results obtained so far are published in [9]. They address tasks of measurements of relevant quantities in 2D histological sections imaged in light microscope. While confirming the importance of the development of automatic quantification methods, in order to overcome problems of high time consumption and subjectivity of manual methods, the obtained results clearly call for further improvements and development.

In this paper we continue the study presented in 9] performed on 2D histologically stained un-decalcified cut and ground sections, with the implant in situ, imaged in light microscope. This technique, so called Exakt technique, [3], is also used for manual analysis. Observations regarding this technique are that 
it does not permit serial sectioning of bone samples with the implant in situ, but on the other hand is the state of the art when implant integration in bone tissue is to be evaluated without, e.g., extracting the device or calcifying the bone. Histological staining and subsequent colour imaging provide a lot of information, where different dyes attach to different structures of the sample, which can, if used properly, significantly contribute to the quality of the analysis results. However, variations in staining and various imaging artifacts are usually unavoidable drawbacks, that make automated quantitative analysis very difficult.

Observing that the measurements obtained by the method suggested in [9], length estimates of bone-implant contact (BIC) in particular, overestimate the manually obtained values (here considered to be the ground-truth), we found the cause of this problem in unsatisfactory segmentation results. Therefore, our main goal in this study is to improve the segmentation. For that purpose, we introduce a novel fuzzy based approach. Fuzzy segmentation methods are nowadays well accepted for handling shading, background variations, and noise/imaging artifacts. We suggest a two-step segmentation method, composed of, first, classification based on discriminant analysis (DA), as a method for automatic seeding required for the second step in the process, fuzzy connectedness (FC). We provide evaluation of the obtained results. The relevant area and length measurements derived from the images segmented by the herein proposed method show higher consistency with the manually obtained ones, compared to those reported in 9 .

The paper is organized as follows: The next section contains a brief description of the previously used method, and some alternatives existing in the literature. Section 3 provides technical data on the used material. In Section 4 the proposed segmentation method is described, whereas in Section 5 we provide results of the automatic quantification and their appropriate evaluation. Section [6] concludes the paper.

\section{Background}

The segmentation method applied in 9] is based on supervised pixel-wise classification [4, utilizing the low intensity of the implant and the colour staining of the bone-region. $R G B$ colour channels are supplemented with saturation $(S)$ and value $(V)$ channels, for improved performance. The pixel values of the three classes present in the images, implant, bone, and soft tissue, are assumed to be multivariate normally distributed. A number of tests carried out confirmed superiority of the approach where the classification is performed in two steps, instead of separating the three classes at the same time. For further details on the method, see [9].

The evaluation of the method exhibits overestimates of the required measurements, apparently caused by not sufficiently good segmentation. We conclude that pixel-wise classification, even though a rather natural choice and frequently used method for segmentation of colour images, relies too much on intensities/colours of individual pixels if used solely; such a method does not exploit spatial information kept in the image. We, therefore, suggest to combine 
spatial and intensity information existing in the image data. In addition, we want to utilize advantages of fuzzy techniques involved in segmentation. Various methods have been developed and exist in the literature; among the most frequently used ones are fuzzy c-means clustering and fuzzy connectedness. Recently, a segmentation method which combines fuzzy connectedness and fuzzy clustering is published, [5]. The method combines spatial and feature space information in the image segmentation process. The proposed algorithm is based on construction of a fuzzy connectedness relation on a membership image, obtained by some (deliberately chosen) fuzzy segmentation method; the suggested one is fuzzy c-means classification.

Motivated by the reasonably good performance of previously explored DA based classification, we suggest another combination of pixel-wise classification and fuzzy connectedness. We extend the crisp DA based classification by introducing an (un)certainty control parameter. We first use this enhanced classification to automatically generate seed regions and, in the second step, the seeded image is segmented by iterative relative FC segmentation method, as suggested in [1]. The method shows improved performance compared to the one in [9].

\section{Material}

Screw-shaped implants of commercially pure titanium were retrieved from rabbit bone after six weeks of integration. This study was approved by the local animal committee at Göteborg University, Sweden. The screws with surrounding bone were processed according to internal standards and guide-lines [7], resulting in $10 \mu \mathrm{m}$ un-decalcified cut and ground sections. The sections were histologically stained prior to light microscopical investigations. The histological staining method used on these sections, i.e. Toluidine blue mixed with pyronin $\mathrm{G}$, results in various shades of purple stained bone tissue: old bone light purple and young bone dark purple. The soft tissue gets a light blue stain. For the suggested method, 1024x1280 24-bit RGB TIFF images were acquired by a camera
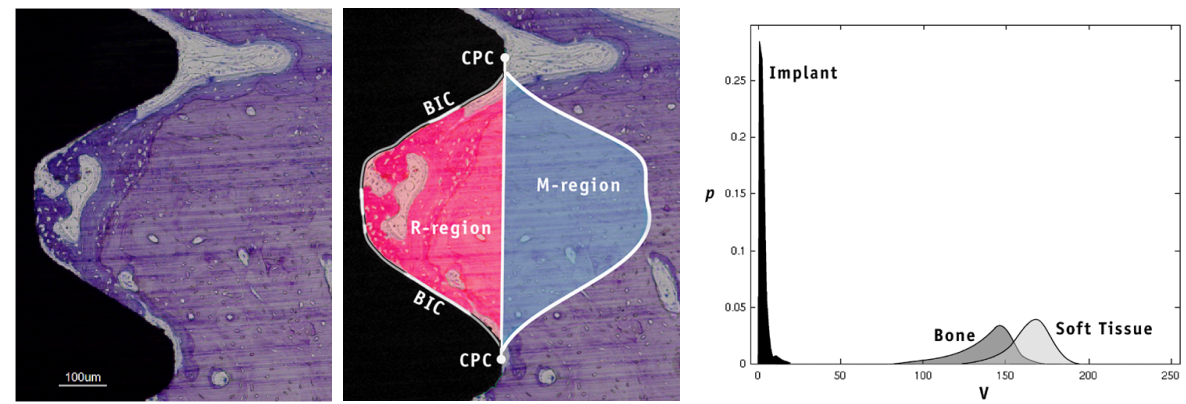

Fig. 1. Left: The screw-shaped implant (black), bone (purple), and soft tissue (light blue) are shown. Middle: Marked regions of interest. Right: Histogram of the pixel distribution in the $V$-channel for a sample image. 
connected to a Nikon Eclipse 80i light microscope. A $10 \times$ ocular was used, giving a pixel size of $0.9 \mu \mathrm{m}$. The regions of interest (ROIs) are marked in Fig. 1 (middle): the gulf between two centre points of the thread crests $(C P C)$ denoted $R$ (reference area); the area $\mathrm{R}$ mirrored with respect to the line connecting the two CPCs, denoted $M$ (mirrored area) and regions where the bone is in contact with the screw, denoted $B I C$. Desired quantifications involve BIC length estimation and areas of different tissues in $\mathrm{R}$ and $\mathrm{M}$; they are calculated for each thread (gulf between two CPCs) expressed as percentage of total length or area 6].

\section{Method}

The main result of this paper is the proposed segmentation method. Its description is given in the first part of this section. In the second part we briefly recall the types of measurements required for quantitative analysis of the bone implant integration.

\subsection{Segmentation}

By pure DA based classification we did not manage to overcome problems originating from artifacts resulting from preparation of specimens (visible stripes after cutting out the slices from the volume), staining of soft tissue that at some places obtained the colour of a bone, and effect of partial coverage of pixels by more than one type of tissue. All this led to unsatisfactory high misclassification rate.

There is a large overlap between the pixel values of the bone and soft tissue classes, as it is visible in the histogram in Figure1 (right). Since all the channels exhibit similar characteristics, a perfect separation of the classes, only based on pixel intensities, is not possible. However, part of the pixels can reliably be classified using a pixel-wise DA approach. We suggest to use the DA classification when certain enough belongingness to a class can be deduced. For the remaining pixels, we suggest to utilize spatial information to address the problem of insufficient separation of the classes in the feature domain.

Automatic Seeding Based on Uncertainty in Classification. Three classes of image pixels are present in the images: implant, bone, and soft tissue. Pixel values are assumed to be multivariate normally distributed. The classification requires prior training; an expert marked different regions using a mouse based interface, after which the $R G B$ values of the regions are stored as a reference.

As in 9], in addition to the three $R G B$ channels, the $S$ and $V$ channels, obtained by a (non-linear) $H S V$ transformation of $R G B$, are also considered in the feature space. For the $H$ channel, it is noticed that it contains considerable amount of noise, and that the classes are not normally distributed, while the distributions of the classes are overlapping to a large extent. For these reasons, the $H$ channel is not considered in the classification.

We introduce a measure of uncertainty in the classification and, with respect to that, an option for pixels to not be classified into any of the classes. A pixels 
may belong to the set $U$ of non-classified (uncertain) pixels due to its low featurebased certainty $u_{F}$, or due to its spatial uncertainty. The set of seed-pixels, $S$, of an image $I$, is then defined as $S=I \backslash U$. They are assigned to appropriate classes in the early stage of the segmentation process. The decision regarding assignment of the elements of the set $U$ is postponed. We define the uncertainty $m_{u}$ of a classification to be $m_{u}=\frac{|U|}{|I|}$, where $|X|$ denotes the cardinality of a set $X$.

To determine feature-based certainty $u_{F}(x)$ of a pixel $x$, we compute posterior probabilities $p_{k}(x)$ for $x$ to belong to each of the observed given classes $C_{k}$. For a multivariate normal distribution, the class-conditional density of an element $x$ and class $C_{k}$ is:

$$
f_{k}(x)=\frac{1}{(2 \pi)^{d / 2}\left|\sum_{k}\right|^{1 / 2}} e^{-\frac{1}{2}\left(x-\mu_{k}\right)^{T} \sum_{k}^{-1}\left(x-\mu_{k}\right)},
$$

where $\mu_{k}$ is the mean value of class $C_{k}, \sum_{k}$ is its covariance matrix, and $d$ is the dimension of the space. Let $P\left(C_{k}\right)$ denote prior probability of a class $C_{k}$. The posterior probability of $x$ to belong to the class $C_{k}$ is then computed as

$$
p_{k}(x)=P\left(C_{k} \mid x\right)=\frac{f_{k}(x) P\left(C_{k}\right)}{\sum_{i} f_{i}(x) P\left(C_{i}\right)} .
$$

To avoid any class bias we assume equal prior probabilities $P\left(C_{k}\right)$.

To generate the sets $S_{k}$ of seed points for each of the classes $C_{k}$, we first perform discriminant analysis based classification in a traditional way and obtain a crisp segmentation of the image into sets $D_{k}$. We initially set $S_{k}=D_{k}$ and then exclude, from each of the sets $S_{k}$, all the points which are considered to be uncertain, regarding belongingness to the class $C_{k}$.

We introduce a measure of feature-based certainty for $x$

$$
u_{F}(x)=\frac{p_{i}(x)}{p_{j}(x)}, \text { for } p_{i}(x)=\max _{k} p_{k}(x) \text { and } p_{j}=\max _{k \neq i} p_{k}(x) .
$$

Instead of assigning pixel $x$ to the class that provides the highest posterior probability, we define a threshold $T_{F}$, and assign the observed pixel $x$ to the component $C_{i}$ only if $u_{F}(x) \geq T_{F}$. Otherwise, $x \in U$, since its probability of belongingness is relatively similar for more than one class, and the pixel is seen as a "border case" in the feature space. Selection of $T_{F}$ is discussed later in the paper. In this way, all the points $x$, having $p_{k}(x)$ as the maximal posterior probability and therefore initially assigned to $S_{k}=D_{k}$, but having $u_{F}(x)<T_{F}$ are in this step excluded form the set $S_{k}$, due to their low feature-based certainty.

Further removal of pixels from $S_{k}$ is performed due to their spatial uncertainty, i.e., their position being close to a border between the classes. To detect such points, we apply erosion by a properly chosen structuring element, SE, to the sets $D_{k}$ separately. The elements that do not belong to the resulting eroded set are removed from $S_{k}$ and added to the set $U$. After this step, all seed points are detected, as $S=\bigcup_{k} S_{k}=I \backslash U$. 
The amount of uncertainty affects the quality of segmentation, as confirmed by the evaluation of the method. We select the value of $m_{u}$, as given by a specific choice of $T_{F}$ and $S E$, according to the results of empirical tests performed.

Iterative Relative Fuzzy Connectedness. We apply iterative relative fuzzy connectedness segmentation method as described in [1]. This version of the fuzzy connectedness segmentation method, originally suggested in [10, is adjusted for segmentation of multiple objects with multiple seeds.

The automatic seeding, performed as the first step of our method, provides multiple seeds for all the (three) existing objects in the image. The formulae for adjacency, affinity, and connectedness relations are, with very small adjustments, taken from [10]. For two pixels, $p, q \in I$, and their image values (intensities) $I(p)$ and $I(q)$, we compute:

Fuzzy adjacency as

$$
\mu_{\alpha}(p, q)=\frac{1}{1+k_{1}\|p-q\|_{2}} \text { for }\|p-q\|_{1} \leq n
$$

$$
\text { Fuzzy affinity as } \quad \mu_{\kappa}(p, q)=\mu_{\alpha}(p, q) \cdot \frac{1}{1+k_{2}\|I(p)-I(q)\|_{2}},
$$

The value of $n$ used in the definition of fuzzy adjacency determines the size of a neighbourhood where pixels are considered to be (to some extent) adjacent. We have tested $n \in\{1,2,3\}$ and concluded that they lead to similar results, and that $n=2$ performs slightly better than the other two tested values. In addition, we use $k_{1}=0$, which leads to the following crisp adjacency relation:

$$
\mu_{\alpha}=\left\{\begin{array}{l}
1, \quad \text { if } \quad\|p-q\|_{1} \leq 2 \\
0, \text { otherwise }
\end{array}\right.
$$

The parameter $k_{2}$, which scales the image intensities and has a very small impact on the performance of $\mathrm{FC}$, is set to 2 .

Algorithm 1, given in [1], is strictly followed in the implementation.

\subsection{Measurements}

The R- and M-regions, as well as the contact line between the implant and the tissue, are defined as described in [9]. The required measurements are: the estimate of the area of bone in $\mathrm{R}$ - and $\mathrm{M}$ - regions, relative to the area of the regions, and the estimate of the BIC length, relative to the length of the border line. Area of an object is estimated by the number of pixels assigned to the object. The length estimation is performed by using Koplowitz and Bruckstein's method for perimeter estimation of digitized planar shapes (the first method of the two presented in [8]). A comparison of the results obtained by the herein proposed method with those presented in [9], as well as with manually obtained ones, is given in the following section. 


\section{$5 \quad$ Evaluation and Results}

The automatic method is applied on three sets of images, each consisting of images of each of the 8 implant threads visible in one histological section. Training data are obtained by manual segmentation of two images from each set. In the evaluation, training images from the set being classified are not included when estimating class means and covariances in a 3 -fold cross-validation fashion.

Our study includes several steps of evaluation: we evaluated the results (i) of the completed segmentation, and (ii) of the quantitative analysis of the implant integration, by comparing relevant measurements with the manually obtained ones, and with the ones obtained in 9. Evaluation of segmentation includes separate evaluation of the automatic seeding and also of the whole two-steps process, i.e., seeding and fuzzy connectedness.

In Figure 2(a) we illustrate the performance of different discriminant analysis approaches in the seeding phase, for different levels of uncertainty $m_{u}$. As the measure of performance, Cohen's kappa, $\kappa$ 2 2 is calculated for the set $S$ and the same part (subset) of the corresponding manually segmented image. We observed two classifiers: linear (LDA), where the covariance matrices of the considered classes are assumed to be equal, and quadratic (QDA), where the

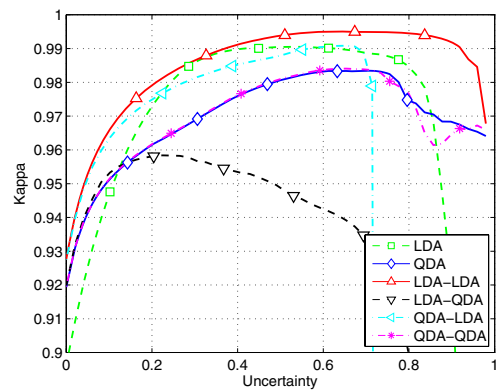

(a)

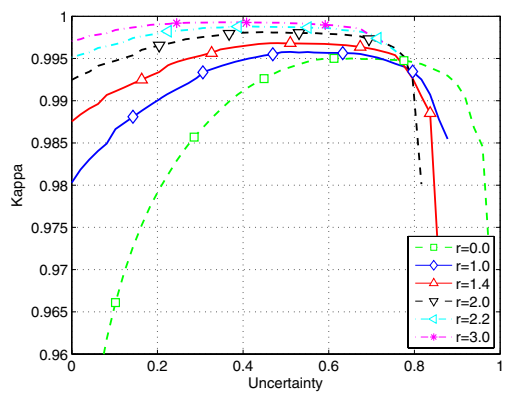

(c)

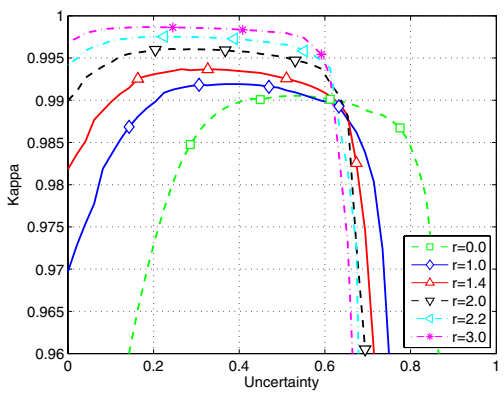

(b)

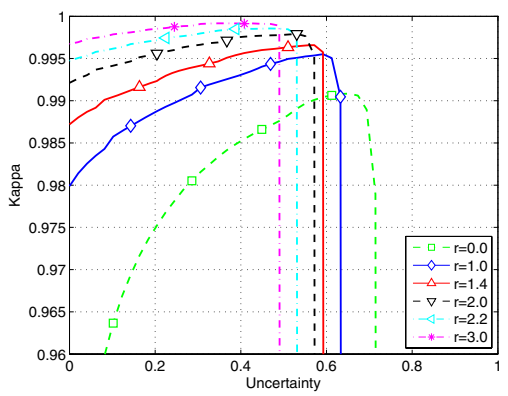

(d)

Fig. 2. Performance of DA. (a) Different DA approaches vs. different levels of $m_{u}$. (b-d) Performance for different radii $r$ of SE, for (b) LDA, (c) LDA-LDA and (d) QDA-LDA. 


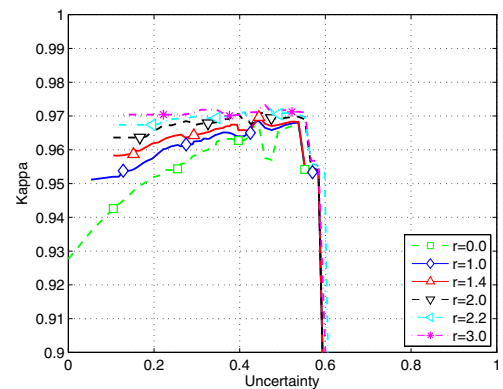

(a)

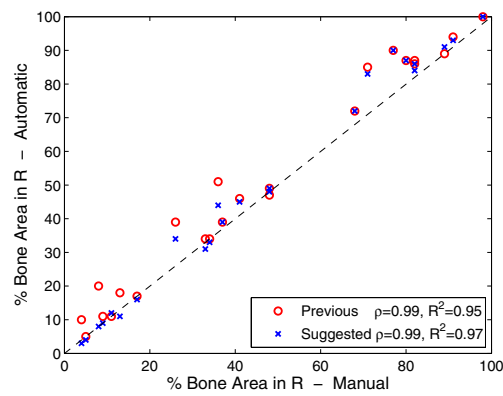

(c)

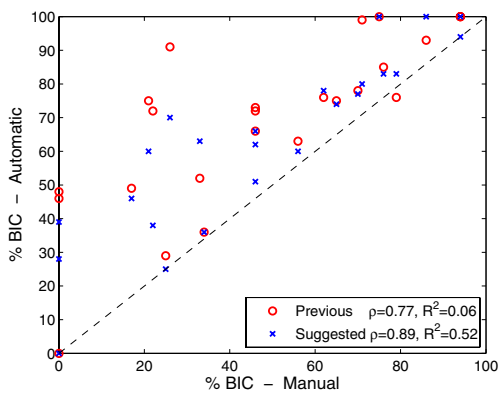

(b)

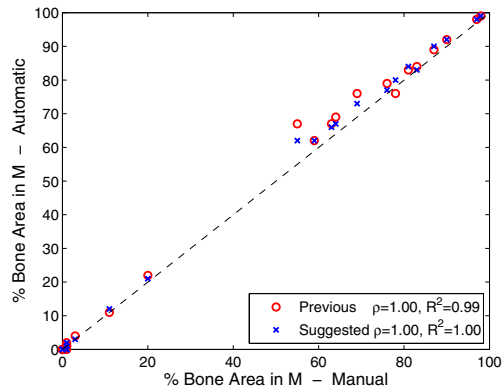

(d)

Fig. 3. Performance of the suggested method. (a) FC from LDA-LDA seeding for different $m_{u}$ and radii $r$ of SE. (b-d) Comparison of measurements from images segmented with the suggested method with those obtained by the method presented in 9 .

covariance matrices of the classes are considered to be different. We observed classification into three classes in one step by both LDA, and QDA, but also by combinations of LDA and QDA used to first classify implant and non-implant regions, and then to separate bone and soft tissue. We notice that three approaches have distinctively better performance than others, for uncertainty up to 0.7 (uncertainty higher than 0.7 leaves, in our opinion, too many non-classified points): LDA-LDA provides the highest values for $\kappa$, while LDA and QDA-LDA perform slightly worse, but good enough to be considered in further evaluation.

Performance of these three DA approaches with respect to different sizes of disk-shaped structuring elements, introducing different levels of spatial uncertainty, is illustrated in Figures 2 (b-d). It is clear that an increase of the size of the structuring elements leads to increased $\kappa$.

Further, we evaluate segmentation results after FC is applied, for different seed images. Figure $3(\mathrm{a})$, shows the performance for LDA-LDA. We see that $\kappa$ increases with increasing size of structuring element, but beyond a radius of $\sqrt{5}$ the improvements are very small. To not loose too much of small structures in the images, we avoid larger structuring elements. 
Important information visible from the plot is the corresponding optimal level of uncertainty to chose. We conclude that uncertainty levels between $25 \%$ and $50 \%$ all provide good results. Segmentations based on seeds from the QDALDA combination show similar behaviour and performance, but exhibiting good performance in a slightly smaller region for $m_{u}$. This robustness of the LDA-LDA combination motivates us to propose that particular combination as the method of choice. The threshold $T_{F}$ can be derived once the size of SE is selected, so that the overall uncertainty $m_{u}$ is at a desired level.

In addition to computing $\mathrm{FC}$ in $R G B$ space, we have also observed $R G B S V$ space, supplied with both Euclidean and Mahalanobis metrics. Due to limited space, we do not present all the plots resulting from this evaluation, but only state that RGBSV space introduces no improvement, neither if Euclidean, nor if Mahalanobis, metric is used. Therefore our further tests include RGB space with Euclidean metrics, as the optimal choice.

Finally, the evaluation of the complete quantification method for bone implant integration is performed based on the required measurements, described in 4.2 The method we suggest is LDA-LDA classification for automatic seeding. Erosion by a disk of radius $\sqrt{5}$ combined with $T_{F}=4$ provides $m_{u} \approx 0.35$. Parameters $k_{1}$ and $k_{2}$ are set to 0 and 2, respectively. Figures 3(b-d) present a comparison of the results obtained by this suggested method with the results presented in [9], and with the manually obtained measurements, which are considered to be the ground truth.

By observing the scatter plots, and additionally, considering correlation coefficients $\rho$ between the respective method and the manual classification, as well as the coefficient of determination $R^{2}$, we conclude that the suggested method provides significant improvement of the accuracy of measurements required for quantitative evaluation of bone implant integration.

\section{Conclusions}

We propose a segmentation method that improves automatic quantitative evaluation of bone implant integration, compared to the previously published results. The suggested method combines discriminant analysis classification, controlled by an introduced uncertainty measure, and fuzzy connectedness segmentation. DA classification is used to define the points which are neither feature-based, nor spatially uncertain. These points are subsequently used as seed-points for the iterative relative fuzzy connectedness algorithm, which assign class belongingness to the remaining points of the images. In this way, both colour information existing in the stained histological material, and spatial information contained in the images, are efficiently utilized for segmentation. The performance of the method provides improved measurements, and overall better automatic quantification of the results obtained in the underlying histomorphometrical study.

The evaluation shows that by the described combination of DA and FC, classification performance measured by Cohen's kappa is increased from $92.7 \%$ to $97.1 \%$, with a corresponding decrease of misclassification rate from $4.8 \%$ to $2.0 \%$, 
as compared to using DA alone. Comparing feature values extracted from the segmented images with manual measurements, we observe an almost perfect match for the bone area measurements, with $R^{2} \geq 0.97$. For the BIC measure, while being significantly better than previously presented method, $R^{2}=0.52$ indicates that further improvements may still be desired. Improvements may possibly be achieved by, e.g., refinement of the affinity relation used in the fuzzy connectedness segmentation, shading correction, appropriate directional filtering, or performing some fuzzy segmentation of the objects in the image, so that more precise measurements can be obtained from the resulting fuzzy representations. Our future work will certainly include some of these issues.

Acknowledgements. Research technicians Petra Hammarström-Johansson, Ann Albrektsson and Maria Hoffman are acknowledged for sample preparations. This work was supported by grants from The Swedish Research Council, 621-2005-3402 and was partly supported by the IA-SFS project RII3-CT-2004506008 of the Framework Programme 6. Nataša Sladoje is supported by the Ministry of Science of the Republic of Serbia through the Projects ON144018 and ON144029 of the Mathematical Institute of the Serbian Academy of Science and Arts. Vladimir Ćurić is supported by the Ministry of Science of the Republic of Serbia through Project ON144016.

\section{References}

1. Ciesielski, K.C., Udupa, J.K., Saha, P.K., Zhuge, Y.: Iterative relative fuzzy connectedness for multiple objects with multiple seeds. Comput. Vis. Image Underst. 107(3), 160-182 (2007)

2. Cohen, J.: A coefficient of agreement for nominal scales. Educational and Psychological Measurement 11, 37-46 (1960)

3. Donath, K.: Die trenn-dunnschliffe-technik zur herstellung histologischer präparate von nicht schneidbaren geweben und materialien. Der Präparator 34, 197-206 (1988)

4. Duda, R.O., Hart, P.E.: Pattern Classification and Scene Analysis. Wiley, New York (1973)

5. Hasanzadeh, M., Kasaei, S., Mohseni, H.: A new fuzzy connectedness relation for image segmentation. In: Proc. of Intern. Conf. on Information and Communication Technologies: From Theory to Applications, pp. 1-6. IEEE Society, Los Alamitos (2008)

6. Johansson, C.: On tissue reactions to metal implants. PhD thesis, Department of Biomaterials / Handicap Research, Göteborg University, Sweden (1991)

7. Johansson, C., Morberg, P.: Importance of ground section thickness for reliable histomorphometrical results. Biomaterials 16, 91-95 (1995)

8. Koplowitz, J., Bruckstein, A.M.: Design of perimeter estimators for digized planar shapes. Trans. on PAMI 11, 611-622 (1989)

9. Sarve, H., Lindblad, J., Johansson, C.B., Borgefors, G., Stenport, V.F.: Quantification of bone remodeling in the proximity of implants. In: Kropatsch, W.G., Kampel, M., Hanbury, A. (eds.) CAIP 2007. LNCS, vol. 4673, pp. 253-260. Springer, Heidelberg (2007)

10. Udupa, J.K., Samarasekera, S.: Fuzzy connectedness and object definition: Theory, algorithms, and applications in image segmentation. Graphical Models and Image Processing 58(3), 246-261 (1996) 